\title{
Stress-Induced Dispersal of Staphylococcus epidermidis Biofilm Is Due to Compositional Changes in Its Biofilm Matrix
}

\author{
Charlène Coulon $^{1}$, Irina Sadovskaya ${ }^{1}$, Philippe Lencel ${ }^{1}$, Saïd Jabbouri ${ }^{2}$, Jeffrey B. Kaplan ${ }^{3}$, \\ Sigrid Flahaut ${ }^{4}$ \\ ${ }^{1}$ UMT, Université du Littoral-Côte d'Opale, Boulogne-Sur-Mer, France \\ ${ }^{2}$ Institut de Recherche pour le Développement, Maadi, Egypt \\ ${ }^{3}$ Department of Oral Biology, New Jersey Dental School, Newark, USA \\ ${ }^{4}$ Service de Microbiologie Appliquée, Université Libre de Bruxelles (ULB), \\ c/o Institut de Recherches Microbiologiques Jean-Marie Wiame (IRMW), Bruxelles, Belgium \\ Email: *coulon0charlene@gmail.com
}

Received October 5, 2012; revised November 3, 2012; accepted November 12, 2012

\begin{abstract}
Biofilm formation is an important virulence factor of Staphylococcus epidermidis. However, little is known about the mechanisms of staphylococcal biofilm dispersal. In the present study, we investigated biofilm dispersal of the model biofilm-forming strain S. epidermidis RP62A under oligotrophic stress conditions. We found that oligotrophic stress led to rapid dispersal of pre-formed biofilms and concomitant changes in the composition of the extracellular matrix, including a decrease in poly- $N$-acetylglucosamine polysaccharide and an increase in proteins. Our results suggest that modifications in biofilm integrity caused by compositional changes in the biofilm matrix can induce biofilm dispersal.
\end{abstract}

Keywords: Staphylococcus epidermidis; Biofilm Composition; Detachment; Nutrient Limitation

\section{Introduction}

Staphylococcus epidermidis is a major cause of indwelling medical device infections [1]. The persistence of such infections is due to the ability of infecting bacteria to adhere to the surface of artificial devices and to form multilayered cell clusters known as biofilms [2]. Adherent or sessile bacteria, embedded in a polymeric biofilm matrix, are more resistant to conventional antimicrobial agents and host defenses than free-living planktonic cells. Biofilm cells are capable of persisting in the presence of antimicrobials at concentrations that are up to 1000 -fold higher than those necessary to eradicate a planktonic population [3]. Biofilm-associated bacteria are difficult to eradicate, and removal of the infected device is often required for an efficient treatment of biofilm infection.

Several studies have investigated the composition of the $S$. epidermidis biofilm matrix, which was found to contain poly- $\beta-(1,6)-N$-acetylglucosamine (Polysaccharide Intercellular Adhesin, PIA) polysaccharide, proteins, and teichoic acids $[4,5]$. PIA, which mediates cell-to-cell adhesion, is considered to be the major functional component in S. epidermidis biofilms [2,6]. Recent data sug-

\footnotetext{
${ }^{*}$ Corresponding author.
}

gest that $S$. epidermidis does not produce enzymes that degrade PIA $[7,8]$. Some $S$. epidermidis strains are able to produce biofilms not containing PIA [9]. However these biofilms are less adherent than PIA-dependent biofilms (unpublished data). PIA-independent biofilm formation was shown to rely on the expression of the accumulation-associated protein (AAP) [10].

The detachment and dispersal of bacterial cells from biofilms is a strategy by which bacteria colonize new niches when space and nutrients become limiting for growth [11]. Nutrient starvation has been shown to induce detachment of biofilms produced by Pseudomonas aeruginosa [12], P. fluorescens [13], P. putida [14] and Aeromonas hydrophila [15]. Glucose depletion can disperse a Staphylococcus aureus biofilm [16]. Jäger et al. [17] showed that $S$. epidermidis biofilms undergo a gradual disintegration over a period of seven-day when challenged with low-glucose medium or phosphate buffered saline. However, none of these studies explain the mechanism of starvation-induced biofilm detachment.

In the present study, we investigated biofilm detachment and the evolution of the biofilm matrix composition under oligotrophic stress conditions in the model strain $S$. epidermidis RP62A. We observed rapid biofilm dispersal and significant changes in the relative amounts of PIA 
and proteins in the biofilm matrix under nutrient starvetion conditions, suggesting that compositional changes in the biofilm matrix can result in biofilm dispersal.

\section{Material and Methods}

\subsection{Bacterial Strains and Media}

S. epidermidis RP62A was kindly provided by Prof. Gerald Pier (Harvard Medical School, Boston, MA). Bacteria were stored at $-20^{\circ} \mathrm{C}$ in $15 \%(\mathrm{v} / \mathrm{v})$ glycerol.

$S$. epidermidis RP62A was cultivated in two different media: 1) an oligotrophic carbon-starved medium (OM) developed in our laboratory: $50 \mathrm{~mL}$ mineral base $\left(2 \mathrm{~g} \cdot \mathrm{L}^{-1}\right.$ glutamic acid, $0.2 \mathrm{~g} \cdot \mathrm{L}^{-1} \mathrm{MgSO}_{4}, 0.01 \mathrm{~g} \cdot \mathrm{L}^{-1} \mathrm{NaCl}, 0.5$ $\left.\mathrm{mg} \cdot \mathrm{L}^{-1} \mathrm{FeCl}_{2}, 1 \mathrm{mg} \cdot \mathrm{L}^{-1} \mathrm{MnSO}_{4}\right), 50 \mathrm{~mL}$ yeast extract $(2$ $\left.\mathrm{g} \cdot \mathrm{L}^{-1}\right), 62.5 \mathrm{~mL}$ acid-hydrolyzed casein $\left(20 \mathrm{~g} \cdot \mathrm{L}^{-1}\right), 5 \mathrm{~mL}$ vitamin $\mathrm{B} 1$ (thiamine; $0.1 \mathrm{mg} \cdot \mathrm{mL}^{-1}$ ), and $832.5 \mathrm{~mL}$ phosphate buffer (100 mM, pH 7); or 2) Tryptic Soy broth (TSB; Becton Dickinson, Le Pont de Claix, France).

\subsection{Growth and Survival Studies}

Bacteria were grown in TSB, supplemented with $1 \%$ glucose $(\mathrm{w} / \mathrm{v})$ for $18 \mathrm{~h}$ at $37^{\circ} \mathrm{C}$ with shaking. Cultures were then diluted at $1 \%$ in fresh TSB or OM medium. The cultures were incubated at $37^{\circ} \mathrm{C}$ with shaking and the absorbance was measured after increasing amounts of time in a He $\lambda$ ios $\beta$ spectrophotometer (Fischer) set to 600 nm.

To study bacterial survival, overnight cultures were diluted in fresh TSB to an $A_{600}$ of 1 . Ten mL of this suspension was harvested by centrifugation at $4000 \times \mathrm{g}$. The pellet was suspended in $50 \mathrm{~mL}$ of fresh medium and incubated at $37^{\circ} \mathrm{C}$ with shaking. At 0,3 and $24 \mathrm{~h}, 500 \mu \mathrm{L}$ of the cell suspension was removed and then serially diluted at $1: 10$ into $0.9 \% \mathrm{NaCl}(\mathrm{w} / \mathrm{v})$. The $10^{-5}, 10^{-6}, 10^{-7}$ dilutions were spread in duplicate onto Tryptic Soy agar plates using $5 \mathrm{~mm}$-diameter glass beads. The plates were incubated for $24 \mathrm{~h}$ at $37^{\circ} \mathrm{C}$, and CFUs were enumerated to calculate the percentage of survival.

\subsection{Biofilm Quantification}

To quantify biofilms, the methods of Christensen et al. [18] with some modifications were used. Briefly, an overnight planktonic culture was diluted 1:100 into fresh TSB medium containing $1 \%$ glucose. Wells of polystyrene tissue culture-treated 96-well microtiter plates (Nunclon, Nunc) were filled with $200 \mu \mathrm{L}$ aliquots of inoculum, and the plate was incubated for $24 \mathrm{~h}$ at $37^{\circ} \mathrm{C}$ with gentle shaking for biofilm formation. After this incubation step, TSB was replaced with fresh TSB or OM medium. After increasing amounts of time, the biofilms were washed twice with $200 \mu \mathrm{L}$ of $0.9 \% \mathrm{NaCl}$ and dried at $55^{\circ} \mathrm{C}$ for 45 min. The adherent cells were stained with $200 \mu \mathrm{L}$ of $0.5 \%$ safranin for $10 \mathrm{~min}$ and rinsed again with $0.9 \%$ $\mathrm{NaCl}$. One hundred $\mu \mathrm{L}$ of $0.9 \% \mathrm{NaCl}$ per well was added, and the absorbance was measured using a $\mu$ Quant microtiter plate reader (Bio-Tek Instruments, Winooski, USA) set to $531 \mathrm{~nm}$. For each experiment, background staining was corrected by subtracting the $A_{531}$ value of safranin bound to non-inoculated controls. Each experiment was performed at least three times.

\subsection{Quantification of PIA and Protein in the Biofilm Matrix}

An overnight planktonic culture was diluted 1:100 into fresh TSB medium containing $1 \%$ glucose. Tissue-culture-treated Petri dishes (100-mm-diam; Greiner) were filled with $20 \mathrm{~mL}$ aliquots of inoculum, and the culture was incubated statically for $24 \mathrm{~h}$ at $37^{\circ} \mathrm{C}$.

For quantification of PIA and protein in the biofilm matrix TSB was removed and replaced by fresh TBS or OM medium. At $0,3,6$ and $24 \mathrm{~h}$, the supernatant was removed, and the biofilm was washed with $5 \mathrm{~mL}$ of $0.9 \%$ $\mathrm{NaCl}$. The biofilm was completely removed from the plastic surface by gentle scraping in the presence of 25 $\mathrm{ng} / \mathrm{mL}$ of the PIA-degrading enzyme dispersin B [19] in $5 \mathrm{~mL}$ of $0.9 \% \mathrm{NaCl}$. The biofilm was then homogenized and aliquots of the suspension were used for CFU enumeration. Cells were then removed from the remaining suspension by centrifugation $\left(5000 \times \mathrm{g}, 10 \mathrm{~min}, 4^{\circ} \mathrm{C}\right)$, and the supernatant was assayed for protein using a BioRad colorimetric assay, and for aminosugars using a Morgan-Elson assay [20], with bovine serum albumin and GlcNAc as standards, respectively.

For studying PIA degradation during an oligotrophic stress, biofilm cells were treated immediately in the control experiment and in biofilm dispersal experiment after $3 \mathrm{~h}$ of incubation at $37^{\circ} \mathrm{C}$. The biofilm was completely removed from the plastic surface by gentle scraping, the culture was transferred into a plastic container, and sonicated on ice (IKASONIC sonicator; 50\% amplitude, cycle $0.5,3$ times for $30 \mathrm{~s}$; IKA Labortechnik, Staufen, Germany). Cells were removed by centrifugation and the supernatant clarified by additional centrifugation (10.000 $\mathrm{g}, 10 \mathrm{~min}, 4^{\circ} \mathrm{C}$ ). The extract was applied to a Sephadex G-50 column $(1 \times 40 \mathrm{~cm})$, eluted with acetic acid $(1 \%)$. Aliquots of each $2 \mathrm{~mL}$ fraction were screened for aminosugars using a Morgan-Elson assay [20], and the corresponding elution profiles were compared.

\section{Results and Discussion}

\subsection{Impact of Oligotrophic Stress on Growth of Staphylococcus epidermidis}

Before we could evaluate the effect of oligotrophic conditions on biofilms, we established the composition of a 
low-carbon medium which does not induce bacterial growth but preserves $100 \%$ survival. We considered that these conditions resulting in impaired growth rate can be defined as an oligotrophic stress. Since $S$. epidermidis is polyauxotrophic, a mineral medium supplemented with minimal amount of yeast and casein extract (oligotrophic medium, OM) was tested. $S$. epidermidis RP62A grown for $24 \mathrm{~h}$ at $37^{\circ} \mathrm{C}$ in $\mathrm{OM}$ showed only few cellular divisions, with maximum growth rate $\mu=0.3$, compared to $\mu=1.46$ in TSB (data not shown). To ensure that bacteria in OM medium were carbon-starved, we added glucose $(0.5 \% \mathrm{w} / \mathrm{v})$ at various times during the first $24 \mathrm{~h}$ of culture. In all cases, the bacterial growth started immediately (data not shown). To evaluate bacterial survival, we quantified the number of CFUs after $0,3,6$ and $24 \mathrm{~h}$ in $\mathrm{OM}$ at $37^{\circ} \mathrm{C}$. The survival rates remained close to $100 \%$. Based on these data, we concluded that bacterial cells in OM medium were stressed by nutrientstarvation, and we tested this effect on S. epidermidis biofilms.

\subsection{Biofilm Detachment by Nutritive Stress}

In order to test the effect of nutritive stress on $S$. epidermidis RP62A biofilms, bacteria were grown in polystyrene 96-well microtiter plates. Once biofilm formed, TSB was replaced with fresh TSB or OM medium and cultures were incubated for $24 \mathrm{~h}$ at $373^{\circ} \mathrm{C}$. The evolution of biofilm was quantified colorimetrically at different incubation times. The results of this experiment clearly showed that the substitution of the rich medium with the oligotrophic one lead to a significant reduction of biofilm (Figure 1(a)), especially during the first $3 \mathrm{~h}$ of nutrient limitation. In the unchanged medium, the amount of biofilm remained stable (Figure 1(a)) indicating that the observed biofilm reduction was not due to turgor, turbulence or shock of replacing media. These results suggest that the nutritive stress caused by the OM medium induced the detachment of $S$. epidermidis RP62A cells from the biofilm.

\subsection{Evolution of Biofilm Matrix Composition under Conditions of Nutrient Stress}

It is known that biofilm matrix of $S$. epidermidis RP62A contains a significant amount of a poly- $\beta-(1,6)-N$-acetylglucosamine polysaccharide (PIA) and proteins $[5,6]$. We measured the amount of aminosugars and proteins in the matrix of $S$. epidermidis RP62A biofilms subjected or not to oligotrophic stress. Biofilms were grown on the surfaces of tissue-culture-treated Petri dishes and subjected to nutritive stress as described above for the 96wells microtiter plates. After increasing amounts of time, biofilm cells were detached, the matrix was homogenized by treatment with dispersin B, and amino-sugars and

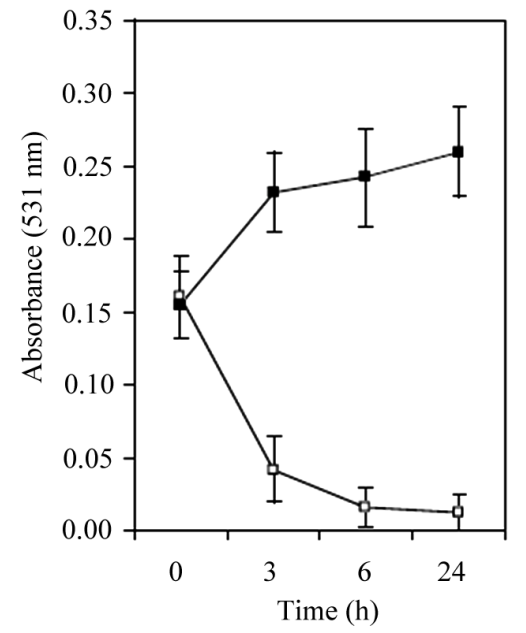

(a)

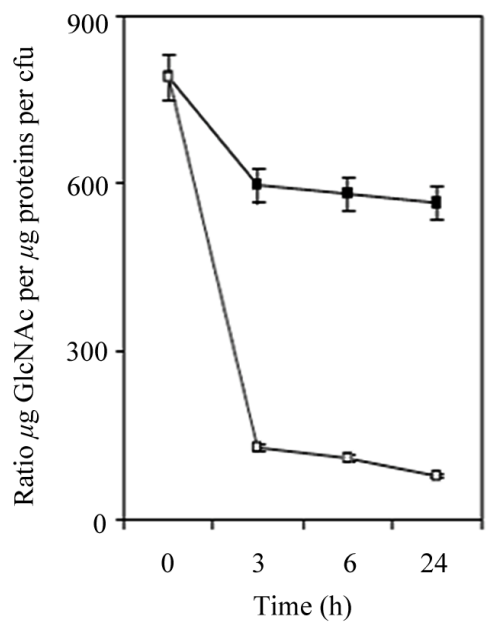

(b)

Figure 1. Oligotrophic stress response of 24-h-old $S$. epidermidis RP62A biofilms. (a) Biofilms were treated with fresh TSB ( $\square$ ) or oligotrophic medium (OM) ( $\square$ ) for 3, 6 or $24 \mathrm{~h}$ at $37^{\circ} \mathrm{C}$ and then rinsed and stained with safranin; (b) Ratio of $\mu \mathrm{g}$ PIA/ $\mu \mathrm{g}$ protein per CFU in TSB (a) and oligotrophic medium (OM) ( $\square)$.

proteins were quantified by colorimetric methods.

Since PIA is considered as the major aminosugar containing component of $S$. epidermidis RP62A biofilm matrix under these experimental conditions, and extracellular teichoic acid (EC TA) contains much smaller amount of GlcNAc [5], we estimated that quantity of aminosugars in biofilm matrix correlates with the quantity of PIA.

Following oligotrophic stress, the amount of aminosugar decreased over time while the amount of protein increased. The ratio of aminosugar to protein (Figure 1(b)) correlated with the amount of biofilm dispersal (Figure 1(a)). In the control without nutritive stress the amounts of aminosugar and protein increased, thus the ratio of aminosugar to protein remained stable over the 


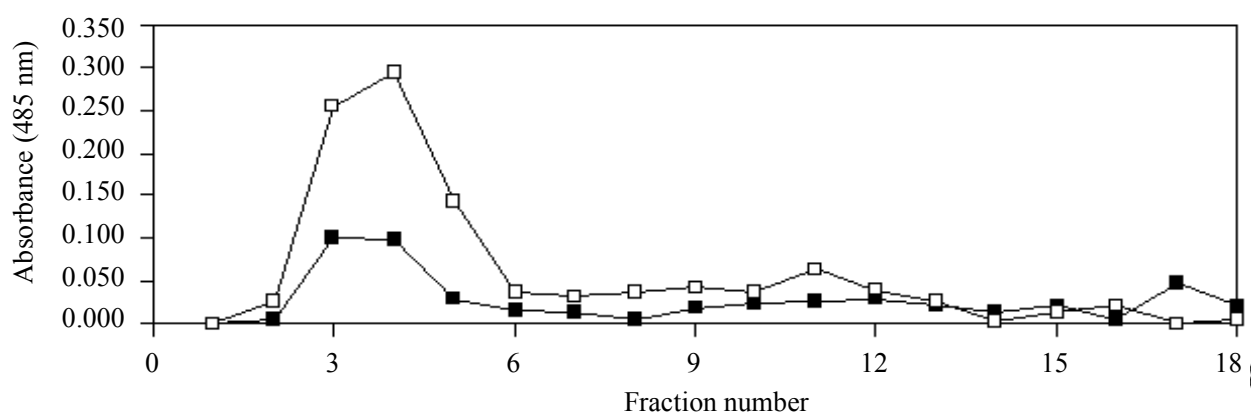

Figure 2. Elution profiles of extracellular extract of $S$. epidermidis RP62A before ( $\square$ ) and after ( $\square$ ) an oligotrophic stress. Aliquots of each $2 \mathrm{~mL}$ fraction were assessed for amino-sugar.

time (Figure 1(b)).

The observed changes in chemical composition of the $S$. epidermidis biofilm matrix under nutrient-limiting conditions are in agreement with previous studies on regulation of biofilm formation by $S$. epidermidis [17]. Activity of the alternative sigma factor $\sigma^{\mathrm{B}}$, the key inductor of genes to adapt to environmental stress, is important for PIA-dependent biofilm formation in S. epidermidis, and $\sigma^{\mathrm{B}}$ mutants do not synthesize PIA, which results in decreased autoaggregation and biofilm formation [21]. It was previously shown that stability of $S$. epidermidis biofilms under glucose-limiting conditions depended on the activity of the alternative sigma factor $\sigma^{\mathrm{B}}$ [17]. Dispersal of the biofilm during nutritive stress, therefore, could be related to regulation in the synthesis of PIA.

In an attempt to better understand the molecular mechanisms of the observed biofilm dispersal, we tested if PIA was degraded during the oligotrophic stress. We compared the chromatographic profiles of the extracellular extract before and after an oligotrophic stress on a Sephadex G-50 gel-filtration column, with the colorimetric screening of fractions for aminosugars. The two elution profiles were similar (Figure 2), indicating that molecular weight (MW) distribution of aminosugar-containing polymers did not change following the oligotrophic stress.

We therefore concluded that the observed biofilm dispersal during an oligotrophic stress was not due to a specific enzymatic degradation of PIA. These data are in agreement with previous studies $[7,8]$ showing that the dispersal of $S$. epidermidis biofilm was not due to a synthesis of a PIA degrading enzyme.

\section{Conclusion}

In conclusion, our findings provide evidence that a decrease in the amount of aminosugar and increase in the amount of protein in the $S$. epidermidis biofilm matrix under nutritive stress conditions can lead to a lower cellto-cell adhesion and thus facilitate a release of bacterial cells into the environment. Understanding mechanisms of biofilm dispersal could lead to the development of clinically useful agents that promote biofilm detachment and thus contribute to a successful treatment of chronic biofilm-related infections.

\section{Acknowledgements}

We are obliged to Dr. Thierry Grard (ULCO) for his supports and fruitful discussion and Prof. Pierre Hardouin (PMOI, ULCO) for his financial support.

\section{REFERENCES}

[1] F. Götz, "Staphylococcus and Biofilms," Molecular Microbiology, Vol. 43, 2002, pp. 1367-1378. doi:10.1046/j.1365-2958.2002.02827.x

[2] D. Mack, A. P. Davies, L. G. Harris, H. Rohde, M. A. Horstkotte and J. K. M. Knobloch, "Microbial Interactions in Staphylococcus epidermidis Biofilms," Analytical and Bioanalytical Chemistry, Vol. 387, No. 2, 2007, pp. 399-408. doi:10.1007/s00216-006-0745-2

[3] N. Cerca, S. Martins, G. B. Pier, R. Oliveira and J. Azeredoa, "The Relationship between Inhibition of Bacterial Adhesion to a Solid Surface by Sub-MICs of Antibiotics and Subsequent Development of a Biofilm," Research in Microbiology, Vol. 156, 2005, pp. 650-655. doi:10.1016/j.resmic.2005.02.004

[4] I. Sadovskaya, P. Chaignon, G. Kogan, A. Chokr, E. Vinogradov and S. Jabbouri, "Carbohydrate-Containing Components of Biofilms Produced in Vitro by Some Staphylococcal Strains Related to Orthopaedic Prosthesis Infections," FEMS Immunology and Medical Microbiology, Vol. 47, 2006, pp. 75-82. doi:10.1111/j.1574-695X.2006.00068.x

[5] I. Sadovskaya, E. Vinogradov, S. Flahaut, G. Kogan and S. Jabbouri, "Extracellular Carbohydrate-Containing Polymers of a Model Biofilm-Producing Strain, Staphylococcus epidermidis RP62A," Infection and Immunity, Vol. 73, No. 5, 2005, pp. 3007-3017. doi:10.1128/IAI.73.5.3007-3017.2005

[6] D. Mack, W. Fischer, A. Krokotsch, K. Leopold, R. Hartmann, H. Egge and R. Laufs, "The Intercellular Adhesin Involved in Biofilm Accumulation of Staphylococcus epidermidis Is a Linear Beta-1,6-Linked Glucosaminoglycan: Purification and Structural Analysis," Journal of Bacteri- 
ology, Vol. 178, No. 1, 1996, pp. 175-183.

[7] M. Otto, "Staphylococcal Biofilms," Current Topics in Microbiology and Immunology, Vol. 322, 2008, pp. 207228.

[8] R. Wang, B. A. Khan, G. Y. Cheung, T. H. Bach, M. Jameson-Lee, K. Kong, S. Y. Queck and M. Otto, "Staphylococcus epidermidis Surfactant Peptides Promote Biofilm Maturation and Dissemination of Biofilm-Associated Infection in Mice," Journal of Clinical Investigation, Vol. 121, No. 1, 2011, pp. 238-248. doi:10.1172/JCI 42520.

[9] G. Kogan, I. Sadovskaya, P. Chaignon, A. Chokr and S. Jabbouri, "Biofilms of Clinical Strains of Staphylococcus that Do Not Contain Polysaccharide Intercellular Adhesin," FEMS Microbiology Letters, Vol. 255, 2006, pp. 1116. doi:10.1111/j.1574-6968.2005.00043.x

[10] H. Rohde, C. Burdelski, K. Bartscht, M. Hussain, F. Buck, M. A. Horstkotte, J. K.-M. Knobloch, C. Heilmann, M. Herrmann and D. Mack, "Induction of Staphylococcus epidermidis Biofilm Formation via Proteolytic Processing of the Accumulation-Associated Protein by Staphylococcal and Host Proteases," Molecular Microbiology, Vol. 55, 2005, pp. 1883-1895. doi:10.1111/j.1365-2958.2005.04515.x

[11] J. B. Kaplan, "Biofilm Dispersal: Mechanisms, Clinical Implications, and Potential Therapeutic Uses," Journal of Dental Research, Vol. 89, No. 3, 2010, pp. 205-218. doi:10.1177/0022034509359403

[12] S. M. Hunt, E. M. Werner, B. Huang, M. A. Hamilton and P. S. Stewart, "Hypothesis for the Role of Nutrient Starvation in Biofilm Detachment," Applied and Environmental Microbiology, Vol. 70, No. 12, 2004, pp. 74187425. doi:10.1128/AEM.70.12.7418-7425.2004

[13] P. J. Delaquis, D. E. Caldwell, J. R. Lawrence and A. R. McCurdy, "Detachment of Pseudomonas fluorescens from Biofilms on Glass Surfaces in Response to Nutrient Stress," Microbial Ecology, Vol. 18, 1989, pp. 199-210. doi:10.1007/BF02075808

[14] M. Gjermansen, M. Nilsson, L. Yang and T. TolkerNielsen, "Characterization of Starvation-Induced Dispersion in Pseudomonas putida Biofilms: Genetic Elements and Molecular Mechanisms," Molecular Microbiology,
Vol. 75, 2010, pp. 815-826. doi:10.1111/j.1365-2958.2009.06793.x

[15] L. K. Sawyer and S. W. Hermanowicz, "Detachment of Biofilm Bacteria Due to Variations in Nutrient Supply," Water Science and Technology, Vol. 37, No.4, 1998, pp. 211-214. doi:10.1016/S0273-1223(98)00108-5

[16] B. R. Boles and A. R. Horswill, "Agr-Mediated Dispersal of Staphylococcus aureus Biofilms," PLoS Pathogens, Vol. 4, 2008, Article ID: e1000052. doi:10.1371/journal.ppat.1000052

[17] S. Jäger, D. Mack, H. Rohde, M. A. Horstkotte and J. K.-M. Knobloch, "Disintegration of Staphylococcus epidermidis Biofilms under Glucose-Limiting Conditions Depends on the Activity of the Alternative Sigma Factor Sigma B," Applied and Environmental Microbiology, Vol. 71, No. 9, 2005, pp. 5577-5581. doi:10.1128/AEM.71.9.5577-5581.2005

[18] G. D. Christensen, W. A. Simpson, J. J. Younger, L. M. Baddour, F. F. Barrett, D. M. Melton and E. H. Beachey, "Adherence of Coagulase-Negative Staphylococci to Plastic Tissue Culture Plates: A Quantitative Model for the Adherence of Staphylococci to Medical Devices," Journal of Clinical Microbiology, Vol. 22, No. 6, 1985, pp. 996-1006.

[19] J. B. Kaplan, C. Ragunath, K. Velliyagounder, D. H. Fine and N. Ramasubbu, "Enzymatic Detachment of Staphylococcus epidermidis Biofilms," Antimicrobial Agents and Chemotherapy, Vol. 48, No. 7, 2004, pp. 2633-2636. doi:10.1128/AAC.48.7.2633-2636.2004

[20] E. Enghofer and H. Kress, "An Evaluation of the MorganElson Assay for 2-Amino-2-Deoxy Sugars," Carbohydrates Research, Vol. 76, 1979, pp. 233-238. doi:10.1016/0008-6215(79)80022-1

[21] S. Jäger, B. Jonas, D. Pfanzelt, M. A. Horstkotte, H. Rohde, D. Mack and J. K. Knobloch, "Regulation of Biofilm Formation by Sigma B Is a Common Mechanism in Staphylococcus epidermidis and Is Not Mediated by Transcriptional Regulation of Sar A," The International Journal of Artificial Organs, Vol. 32, No. 9, 2009, pp. 584-591. 